\title{
A Ground-Based Interferometric Phased Array Trigger for Ultra-high Energy Neutrinos
}

\author{
A. G. Vieregg ${ }^{* 1}$, P. Allison ${ }^{2}$, C. Deaconu ${ }^{1}$, M. DuVernois ${ }^{3}$, E. Oberla ${ }^{1}$, and S. Wissel ${ }^{4}$ \\ for the Askaryan Radio Array (ARA) Collaboration,
}

K. Bechtol ${ }^{5}$, M. Bogdan ${ }^{1}$, A. Ludwig ${ }^{1}$, M. Ransom ${ }^{1}$, A. Romero-Wolf ${ }^{6}$, and K. Wei ${ }^{1}$.

Full author list: http://ara.wipac.wisc.edu/collaboration/authors/ara_icrc17

${ }^{1}$ University of Chicago,

${ }^{2}$ Ohio State University,

${ }^{3}$ University of Wisconsin, Madison,

${ }^{4}$ California Polytechnic State University, San Luis Obispo,

${ }^{5}$ Large Synoptic Survey Telescope (LSST),

${ }^{6}$ Jet Propulsion Laboratory,

E-mail: avieregg@kicp.uchicago.edu

\begin{abstract}
We are developing a ground-based radio interferometric phased array for radio detection of high energy neutrinos, in an effort to lower the energy threshold of radio detection experiments while increasing the effective volume at high energies. The radio detection technique looks for Askaryan emission from neutrinos interacting in large volumes of glacial ice. The principle behind the phased array technique is coherent summing of the broadband, impulsive Askaryan signal from multiple antenna channels, increasing the signal-to-noise ratio for triggering on weak signals. We first discuss simulations and validation measurements related to the phased array technique, including results from a preliminary Monte Carlo simulation, a demonstration of beamforming and measurements of thermal noise correlation in an anechoic chamber, and results from a trigger simulation. We then discuss the design and development of the first ground-based interferometric phased array trigger system, a 16-channel system that has been built and will be deployed as part of one Askaryan Radio Array (ARA) station in December 2017 at the South Pole.
\end{abstract}

35th International Cosmic Ray Conference - ICRC2017

10-20 July, 2017

Bexco, Busan, Korea

\footnotetext{
* Speaker.
} 


\section{Introduction}

Cosmogenic ultra-high energy (UHE) neutrinos $\left(E>10^{17} \mathrm{eV}\right)$ still await discovery. A UHE neutrino observatory would determine the origin of the highest energy cosmic rays and study the evolution of the highest energy sources. UHE neutrinos are made as a byproduct of the GZK process, the interaction of UHE cosmic rays with the cosmic microwave background $[1,2,3]$. The IceCube experiment has also detected a separate population of astrophysical neutrinos with energies up to $\sim 10 \mathrm{PeV}[4,5]$. The source of these astrophysical neutrinos is unknown and the spectral index is uncertain [6].

One promising method for detecting high energy neutrinos is via the Askaryan effect - the coherent, impulsive radio emission from electromagnetic showers induced by neutrinos in a dielectric [7]. For high energy showers, the long-wavelength radio emission dominates. Detecting a significant rate of high energy astrophysical and cosmogenic neutrinos requires a large volume of a dielectric material with a long radio attenuation length $\left(L_{\alpha}>1 \mathrm{~km}\right)$, such as glacial ice. The Antarctic Impulsive Transient Antenna (ANITA) long duration balloon experiment currently holds the best constraints on the flux of neutrinos above $10^{19.5} \mathrm{eV}$ [8]. The ground-based Askaryan Radio Array (ARA) and Antarctic Ross Ice-Shelf Antenna Neutrino Array (ARIANNA) experiments are under development in Antarctica and have energy thresholds $\gtrsim 50 \mathrm{PeV}$, probing the heart of the cosmogenic neutrino regime $[10,11]$.

\subsection{The Ground-Based Interferometric Phased Array Concept}

We are developing a ground-based interferometric phased array detector, and will deploy the first example of such a trigger as part of the ARA experiment at the South Pole in December 2017. This type of trigger has the potential to push the energy threshold for radio detection down compared to currently-deployed detector configurations, further probing the astrophysical neutrino flux observed by IceCube while also increasing acceptance at higher energies.

We coherently add signals from multiple low-gain antennas with the proper time delays to account for distances between antennas, effectively increasing the gain of the system of antennas for incoming plane waves from a given direction. Incident plane wave signals such as neutrino signals add coherently, while thermal noise adds incoherently, increasing the signal-to-noise ratio (SNR) of the output signal for neutrino events. Many different sets of delays of signals from the same antennas create multiple effective antenna beam patterns that together cover the same solid angle as each individual antenna but with much higher gain. See Reference [12] for more details.

Table 1 shows results from a preliminary Monte Carlo simulation for an ideal detector, described in Reference [12]. The Table shows cumulative relative expected event rates for three different experimental configurations (16-antenna stations, 16-antenna stations with a phased array trigger, and 400-antenna stations with a phased-array trigger) over three years for ten independent stations installed $100 \mathrm{~m}$ below the surface at Summit Station, Greenland for a variety of astrophysical and cosmogenic models. Summit Station is an appealing site for radio detection of UHE neutrinos, with comparable ice depth to South Pole, shallower firn, but shorter attenuation length [13]. The models shown for astrophysical neutrinos are the best-fit spectrum $\left(E^{-2.5}\right)$ from a combined global analysis of IceCube data [6] and the best-fit spectrum $\left(E^{-2.1}\right)$ from an analysis of up-going muon events [14]. We also show results for two cosmogenic neutrino models [15]. There is signifi- 


\begin{tabular}{|l|c|c|c|c|}
\hline Station Configuration & $\begin{array}{c}\text { Power Law } \\
\left(E^{-2.5}\right)\end{array}$ & $\begin{array}{c}\text { Power Law } \\
\left(E^{-2.1}\right)\end{array}$ & $\begin{array}{c}\text { Optimistic } \\
\text { Cosmogenic }\end{array}$ & $\begin{array}{c}\text { Pessimistic } \\
\text { Cosmogenic }\end{array}$ \\
\hline 16-antenna & 1.0 & 10.9 & 7.7 & 2.3 \\
16-antenna, phased & 5.3 & 33.0 & 19.6 & 6.0 \\
400-antenna, phased & 34.4 & 114.8 & 52.9 & 15.6 \\
\hline
\end{tabular}

Table 1: Expectation values for the total number of triggered events in three years for 10 independent stations installed $100 \mathrm{~m}$ below the surface at Summit Station, Greenland, in different configurations (16-antenna stations, 16-antenna stations with a phased array trigger, and 400-antenna stations with a phased-array trigger) for spectra based on recent IceCube analyses $[6,14]$ and for optimistic and pessimistic cosmogenic neutrino models [15].

cant improvement, especially at low energies, by moving to an interferometric phased array trigger scheme.

In Section 2.1, we describe measurements made in an anechoic chamber to validate the beamforming technique and measure correlation of thermal noise between adjacent antennas. Section 2.2 describes a comparison between measurements in the anechoic chamber and a trigger simulation. In Section 3, we describe the 16-channel interferometric phased array system that we have designed and built, and which will be deployed as part of the ARA experiment in late 2017. We conclude in Section 4.

\section{Validation Measurements in an Anechoic Chamber}

We have performed tests in an anechoic chamber to validate the phased array technique, including a validation of the beamforming technique, measurements of thermal noise correlation between adjacent antennas, and trigger studies, all of which are described in this Section. The test system consisted of two or three antennas laid out end-to-end inside the anechoic chamber, with each antenna in its neighbor's null. Signals from each antenna were amplified by a dual-stage frontend amplifier chain, band-pass filtered, and read out using an oscilloscope. For the beamforming validation and trigger studies measurements, we set up a transmitting antenna $4 \mathrm{~m}$ away from the receiving antennas inside the chamber. The walls of the anechoic chamber were between $1 \mathrm{~m}$ and $3 \mathrm{~m}$ from the antennas. The results of these tests are described in detail in Reference [16].

\subsection{Beamforming and Thermal Noise Correlation}

Figure 1 shows the result of a test of beamforming of impulsive signals for low-gain, broadband antennas placed end-to-end in an anechoic chamber. The left-hand panel shows the impulsive signal received in the two independent antenna channels. Each channel had a slightly different impulse response, gain, and noise figure, dominated by differences between individual antennas and front-end low-noise amplifiers. The middle panel shows the beam that we formed in hardware (using splitters and combiners), compared to the ideal beam that should be formed, calculated in analysis.

Since the power of the interferometric trigger relies on the fact that thermal noise is uncorrelated between antennas, and beamforming to cover the entire available solid angle requires less 

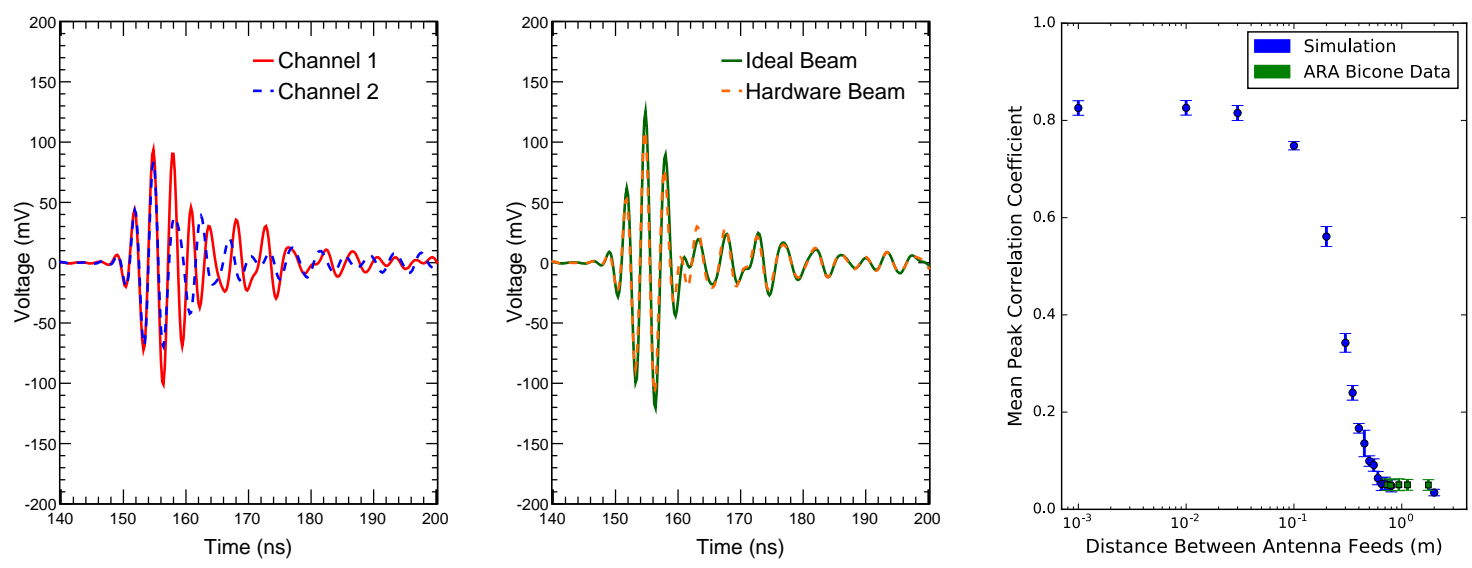

Figure 1: Left: An overlay of waveforms recorded in an anechoic chamber, averaged over 500 events, for two channels of antennas located end-to-end, when transmitting a fast impulse to the antennas. Middle: An overlay of waveforms, averaged over 500 events, for the hardware-summed beam and the coherent waveform calculated directly from the averaged waveforms shown on the left. Right: Results of a thermal noise simulation compared to measured noise correlation as a function of feed-to-feed antenna spacing. Shown is the peak cross-correlation coefficient averaged over 500 simulated or measured events in a \pm 6.0 ns time window. The data goes to the smallest physically-allowed spacing $(76 \mathrm{~cm})$. These tests use the broadband bicone antennas used by ARA (150-850 MHz). This Figure is modified from Reference [16].

computation the closer the antennas are, we measured the thermal noise correlation between adjacent low-gain antennas placed in each other's nulls (end-to-end) in an anechoic chamber and compared it to a Monte Carlo simulation of thermal noise. We changed the spacing between the antennas, ranging from as close as physically possible to a distance of over $1.5 \mathrm{~m}$ between antenna feeds, to measure the level of correlated noise between channels as a function of the distance between the antennas. The right panel of Figure 1 shows the result of our measurements for the broadband bicone antennas used by ARA $(150-850 \mathrm{MHz})$. When the antennas were placed as close as physically possible (corresponding to a feed-to-feed spacing of as small as $76 \mathrm{~cm}$ ), there was no observed correlation, consistent with simulations.

In addition, we have made measurements at Summit Station, Greenland, which show that the noise observed $100 \mathrm{~m}$ below the surface is consistent with absolute thermal noise levels, a thermal noise spectrum, and that noise between adjacent channels is uncorrelated [16].

\subsection{Trigger Studies}

We measured event rates from a 3-antenna array in the anechoic chamber while sending impulsive signals from the transmitting antenna at several attenuation levels to measure the expected trigger efficiency for a phased array. The left-hand plot in Figure 2 shows the measured trigger efficiency at a trigger rate of $100 \mathrm{~Hz}$ using an 8-sample averaging interval. These measurements compare well with simulation results shown by the solid lines in the left-hand plot of Figure 2. We then extrapolate the simulation to larger phased arrays, shown on the right-hand plot in Figure 2. A 16-antenna linear phased array that is set to trigger on impulsive events in a single beam at 100 

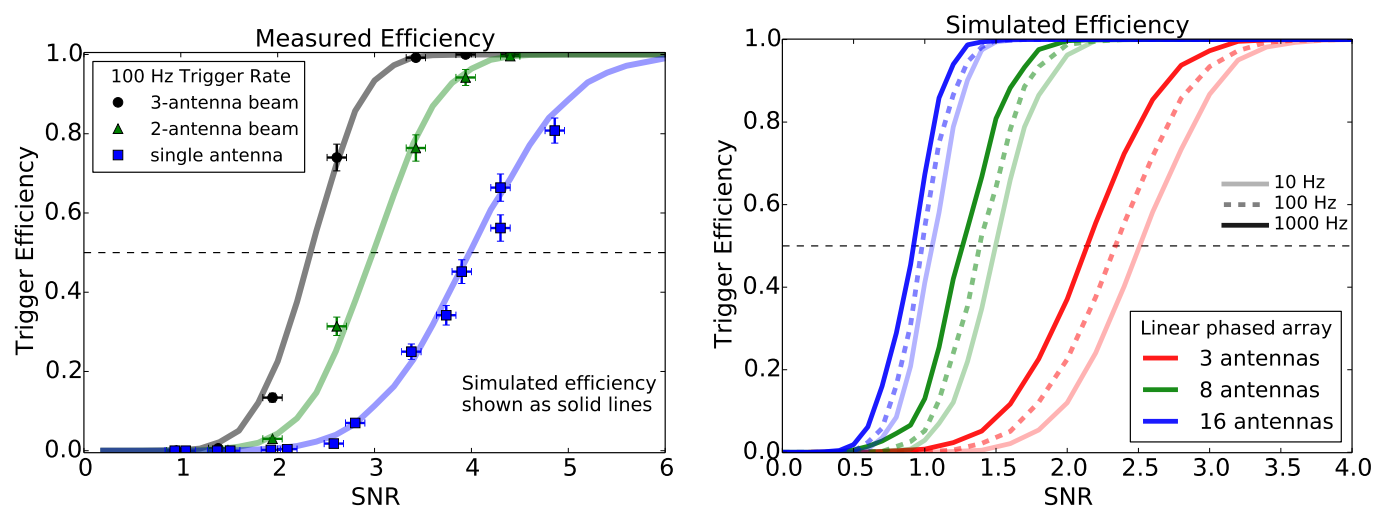

Figure 2: Trigger efficiency vs. SNR. The left plot shows anechoic chamber results from a 3-antenna array (data points) compared to a simulation of uncorrelated noise added to the system impulse response (lines). The data points are taken from anechoic chamber measurements with a fast impulsive signal. The right plot shows the simulated trigger efficiencies for 3, 8, and 16-antenna broadband phased arrays in a single formed beam. Efficiency curves are drawn for per-beam trigger rates of $1 \mathrm{kHz}, 100 \mathrm{~Hz}$, and $10 \mathrm{~Hz}$. This Figure is from Reference [16].

$\mathrm{Hz}$ achieves a $50 \%$ efficiency at a signal-to-noise ratio (SNR) of $\sim 1$, compared to single-antenna trigger thresholds, which reach 50\% efficiency at an SNR of $\sim 4$ at the same trigger threshold.

\section{An Interferometric Phased Array Trigger for ARA}

We are currently constructing a 16-channel interferometric trigger that we will deploy as part of one station of ARA (ARA-5) at the South Pole in December 2017. A schematic of the ARA station that will include the interferometric phased array trigger is shown in Figure 3. We will deploy 16 antennas, spaced $1 \mathrm{~m}$ apart, on a single string down a borehole at the center of ARA5. We will perform the beamforming on the phased array trigger board, which sits at the surface, and then send a trigger signal to the main ARA data acquisition system (DAQ), along with other information about each triggered event, including event waveforms. The ARA station itself has 16 antenna channels, which will be digitized and recorded by the main ARA DAQ along with the waveforms from the phased array trigger string. The radio frequency (RF) front-end design of the phased array string will be similar to that of the rest of ARA (including antennas, amplifiers, filters, and cabling), but mechanical and power system modifications are required to deploy 16 antennas on one string (rather than the 4 per string previously deployed by ARA).

\subsection{Trigger System Design and In-Lab Performance}

We designed an 8-channel digitizer board to perform real-time correlations between the RF signal inputs in order to form a directional trigger for a radio array. This board, shown in Figure 4, was made using off-the-shelf components including a 7-bit, 1.5 GSa/sec ADC from Texas Instruments (ADC07C1520) and a high performance Altera (Intel) Arria V FPGA. An on-board microcontroller board allows communication with a fast Ethernet link and includes $32 \mathrm{MB}$ of SDRAM. The board consumes roughly $25 \mathrm{~W}$. 


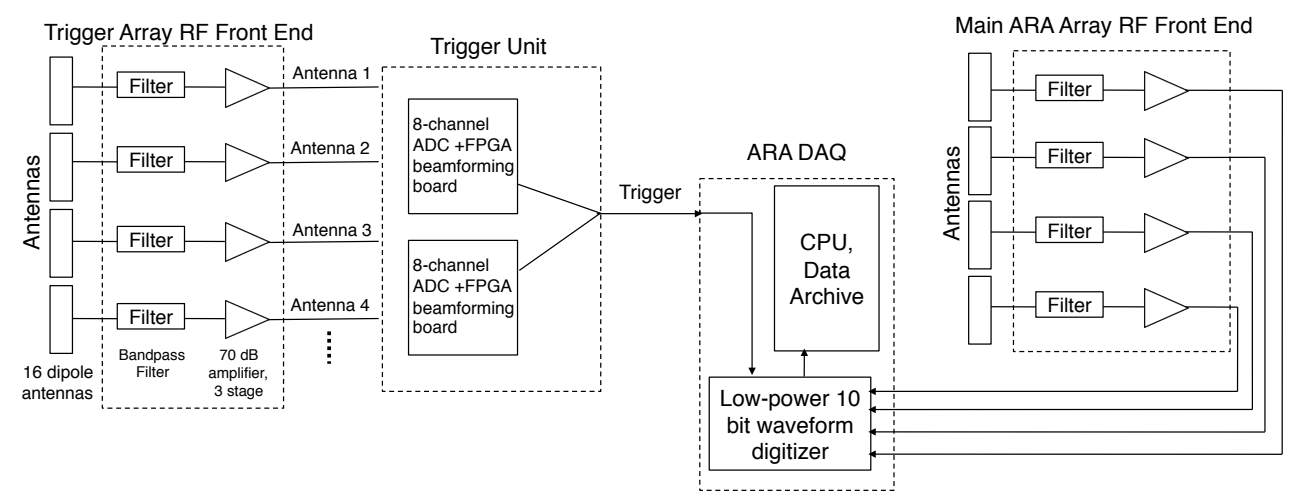

Figure 3: A schematic of the 16-antenna phased array trigger that will be deployed as part of an ARA station (ARA-5) in December 2017 at the South Pole. For simplicity, not all channel paths are depicted.
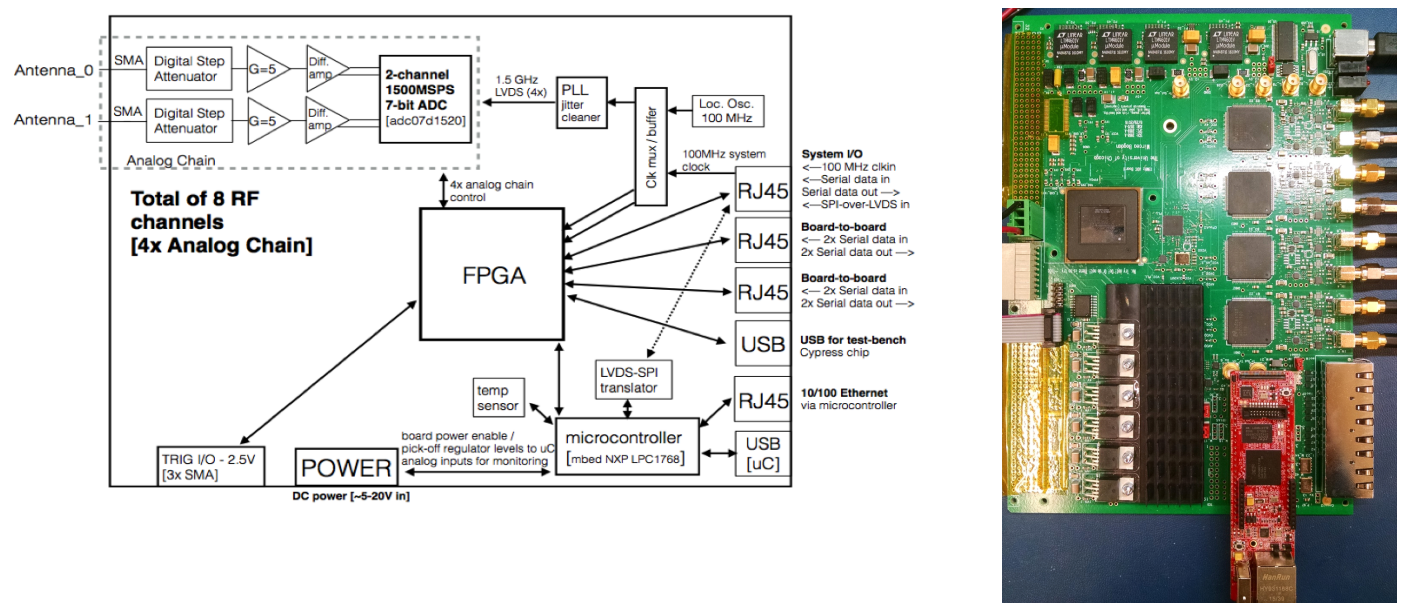

Figure 4: Left: A schematic of the 8-channel trigger board developed for first use in the ARA experiment at the South Pole. Right: A picture of one board, designed and fabricated at the University of Chicago.

Two of these boards will be deployed to the South Pole in 2017, comprising two adjacent 8 -channel phased arrays. Figure 5 shows the result of a simulation of the beams from this trigger system and the antenna layout we will deploy (16 antennas spaced $1 \mathrm{~m}$ apart on a single string down a single borehole). We achieve an $11 \mathrm{dBi}$ gain in each primary beam, and beams overlap close to their $3 \mathrm{~dB}$ point. We can fill in gaps in solid angle with lower-gain beams from 4 antennas (secondary beams), that we can steer to cover angles between primary beams.

The board performs dead time-less digitization with beamforming and power calculations done on the FPGA. We reduce the input dynamic range to 4 bits of the available 7 ADC bits in order to reduce the processing overhead in the FPGA. The response and basic functionality of the board, in a noiseless environment, is shown in Figure 6. The time delay-and-sum technique, in which an 

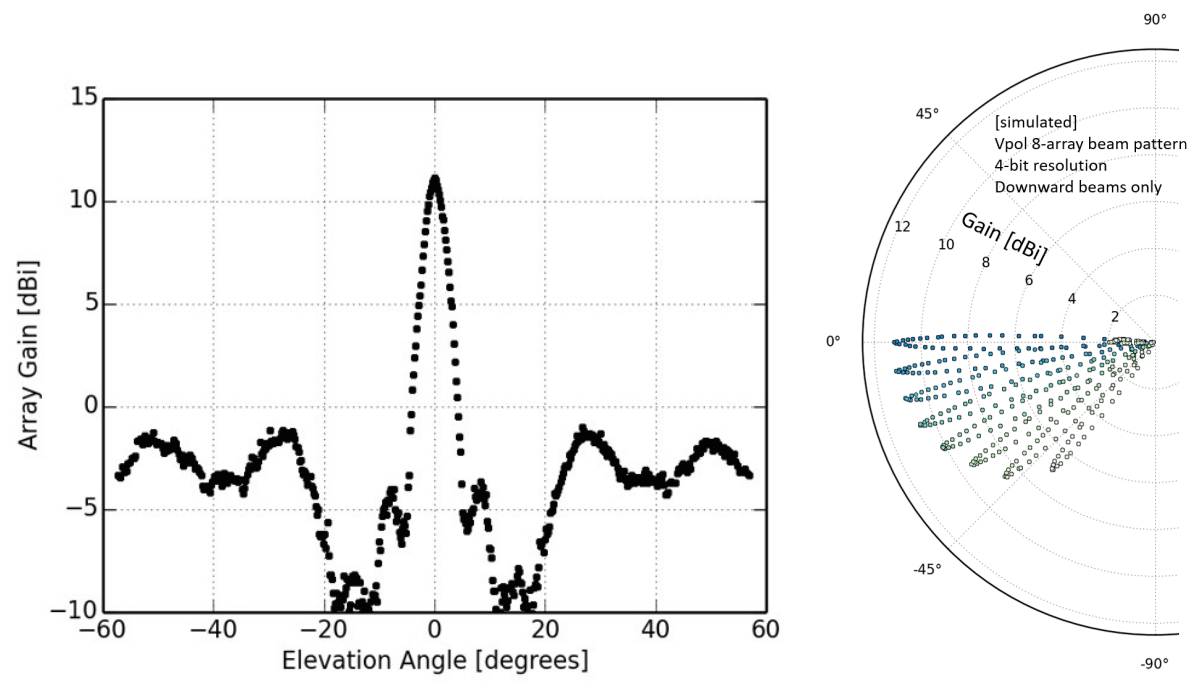

Figure 5: Left: The gain of the 8-antenna beam formed using ARA bicone antennas and the phased array trigger string as a function of elevation. The width of the beam is determined by the frequency response and the spacing of the antennas. The azimuthal response is uniform. Right: The spacing (in elevation) and gain of adjacent beams formed using the phased array trigger system. The spacing of the primary beams is set by the sampling rate of the digitizers, which is $1.5 \mathrm{GSa} / \mathrm{sec}$. We need 8 primary beams to cover the solid angle of interest (full azimuthal coverage and horizontal to $-45^{\circ}$ in elevation).

integer number of sample delays are applied to each channel and a sum is calculated for each set of delays that correspond to an physical input plane-wave direction, is used to perform beamforming. In the example shown in Figure 6, the peak beam is found at zero relative delay between the input signals, as is expected with the input waveforms shown. Finally, the transient power is calculated in each beam, which can be compared to a threshold level for triggering.

\section{Conclusions}

The ground-based phased array for detection of high-energy neutrinos is a promising technique. After a series of simulations and validation measurements [16], we are designing and constructing such a trigger to be implemented at the South Pole by the ARA experiment. The phased array trigger is expected to increase the event rate of an ARA station at the trigger level by up to a factor of $\sim 5$ at $10 \mathrm{PeV}$ and up to a factor of $\sim 3$ at $100 \mathrm{PeV}$. Anechoic chamber measurements with phased antennas are consistent with the expected improvement in SNR and with uncorrelated noise between channels. We have fabricated trigger boards and demonstrated initial beamforming for the system that ARA will deploy in late 2017. Pending successful demonstration of the technique, a larger array with hundreds of antennas per station could be proposed either at the South Pole or in Greenland to achieve a low energy threshold capable of providing significant overlap in energy with IceCube in the PeV energy range, and extending the measurement of high energy neutrinos through the higher-energy cosmogenic neutrino range. 

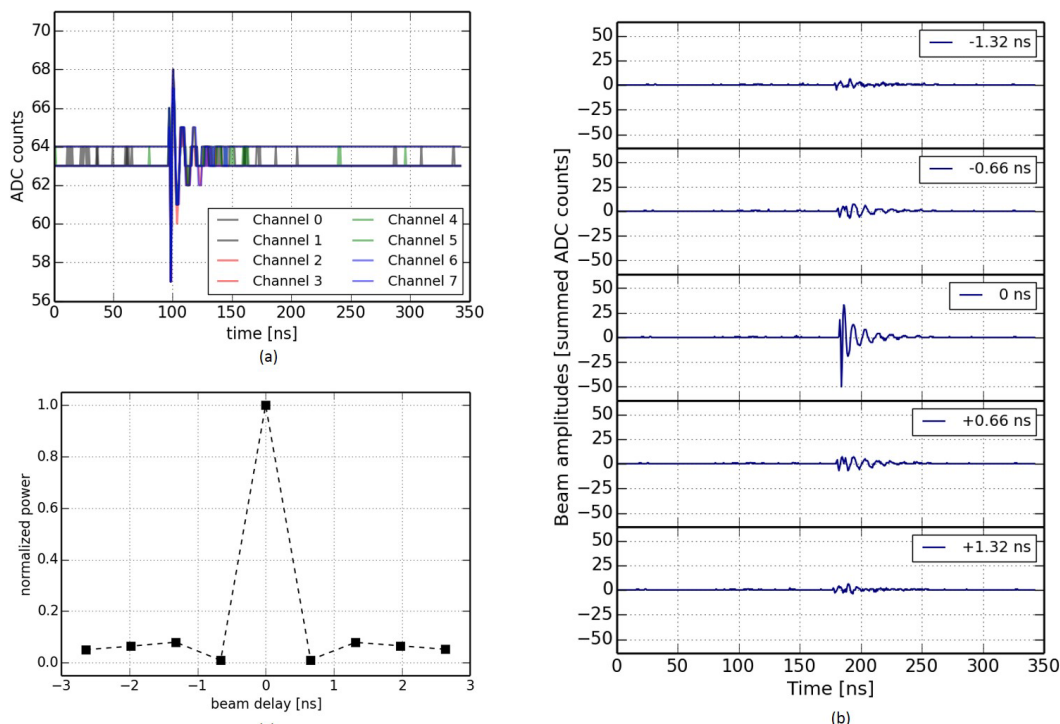

(c)

Figure 6: Waveform acquisition and FPGA-based beamforming using the phased array electronics. (a) We split and injected a band-limited impulse on each of the 8 channels. The input signal dynamic range is limited to 4 bits out of the 7 bits available on the ADC chip. (b) Five of the central pointing beams, formed using delay-and-sum beamforming on the FPGA. (c) Maximum transient power in each beam as calculated on the FPGA.

\section{References}

[1] K. Greisen, Phys. Rev. Lett. 16, 748-750 (1966).

[2] G. Zatsepin and V. Kuzmin, JETP Lett. 4, 78 (1966).

[3] V. Beresinsky and G. Zatsepin, Phys. Lett. B. 28, 423-424 (1969).

[4] IceCube Collaboration, M. Aartsen et al., Phys. Rev. Lett. 113:101101 (2014).

[5] IceCube Collaboration, M. Aartsen et al., Phys. Rev. Lett. 111:021103 (2013).

[6] IceCube Collaboration, M. Aartsen et al., Ap. J. 809:98 (2015).

[7] G. Askaryan, JETP 14:2, 441-443 (1962).

[8] ANITA Collaboration, P. Gorham et al., Phys. Rev. D. 82:022004 (2010).

[9] P. Gorham et al., Astroparticle Phys. 35, 242-256 (2011).

[10] ARA Collaboration, P. Alliston et al., Phys. Rev. D. 93:082003 (2016).

[11] S. Barwick et al., Astroparticle Phys. 70, 12-26 (2015).

[12] A. Vieregg, K. Bechtol, and A. Romero-Wolf, JCAP 2, 005 (2016).

[13] J. Avva et al., J. Glaciology, 61:1005-1011 (2015).

[14] IceCube Collaboration, S. Schoenen et al., Proc. of Science, ICRC 2015 (2015).

[15] K. Kotera, D. Allard, and A. Olinto, JCAP 10:013 (2010).

[16] J. Avva et al., arXiv:1605.03525 (2016). 